\title{
BMJ Open Attitudes, risk of infection and behaviours in the operating room (the ARIBO Project): a prospective, cross-sectional study
}

\author{
Gabriel Birgand, ${ }^{1,2,3}$ Christine Azevedo, ${ }^{4,5}$ Gaelle Toupet, ${ }^{3}$ Roger Pissard-Gibollet, ${ }^{5}$ \\ Bruno Grandbastien, ${ }^{6}$ Eric Fleury, ${ }^{5,7}$ Jean-Christophe Lucet ${ }^{1,2,3}$
}

To cite: Birgand $\mathrm{G}$,

Azevedo C, Toupet G, et al. Attitudes, risk of infection and behaviours in the operating room (the ARIBO Project): a prospective, crosssectional study. BMJ Open 2014;4:e004274.

doi:10.1136/bmjopen-2013004274

- Prepublication history for this paper is available online. To view these files please visit the journal online (http://dx.doi.org/10.1136/ bmjopen-2013-004274).

Received 17 October 2013 Revised 27 November 2013 Accepted 29 November 2013

CrossMark

For numbered affiliations see end of article.

Correspondence to Dr Gabriel Birgand; gbirgand@gmail.com

\section{ABSTRACT}

Introduction: Inappropriate staff behaviours can lead to environmental contamination in the operating room $(\mathrm{OR})$ and subsequent surgical site infection (SSI). This study will focus on the continued assessment of $O R$ staff behaviours using a motion tracking system and their impact on the SSI risk during surgical procedures.

Methods and analysis: This multicentre prospective cross-sectional study will include 10 ORs of cardiac and orthopaedic surgery in 12 healthcare facilities (HCFs). The staff behaviour will be assessed by an objective, continued and prolonged quantification of movements within the OR. A motion tracking system including eight optical cameras (VICON-Bonita) will record the movements of reflective markers placed on the surgical caps/hoods of each person entering the room. Different configurations of markers positioning will be used to distinguish between the staff category. Doors opening will be observed by means of wireless inertial sensors fixed on the doors and synchronised with the motion tracking system. We will collect information on the OR staff, surgical procedures and surgical environment characteristics. The behavioural data obtained will be compared (1) to the 'best behaviour rules' in the OR, pre-established using a Delphi method and (2) to surrogates of the infectious risk represented by microbiological air counts, particle counts, and a bacteriological sample of the wound at closing. Statistics will be performed using univariate and multivariate analysis to adjust on the aerolic and architectural characteristics of the $\mathrm{OR}$. A multilevel model will allow including surgical specialty and HCFs effects. Through this study, we will develop an original approach using high technology tools associated to data processing techniques to evaluate 'automatically' the behavioural dynamics of the OR staff and their impact on the SSI risk.

Ethics and dissemination: Approbation of the Institutional Review Board of Paris North Hospitals, Paris 7 University, AP-HP (no 11-113, 6 April 2012). The findings will be disseminated through peerreviewed journals, and national and international conference presentations.

\section{Strengths and limitations of this study}

- This study will be the largest performed on the topic. Twenty operating room (OR) in 12 healthcare facilities will be included and will describe a large panel of practices in cardiac and orthopaedic surgery. The second originality will be the use of high-technology tracking systems. This new technology device will allow obtaining full and systematic data impossible to collect by human observers. Finally, the behavioural aspect will be approached by several ways with the interpretation of observations by psychology specialists and the perception of surgical staff. The comparison of the perceptions and practices will allow in understanding the behaviours. These data will orient through solutions to improve practices.

- The study will present several limitations. The first will be the reluctance to participate and the modification of behaviour due to the presence of video camera in the $\mathrm{OR}$. This bias will be managed by a large communication to all the surgical staff before the beginning of the study in each centre. We will insist on the fact that all the data will be anonymous using presentation of the system and simulations. The motion capture system will not record videos but will only keep the positions of the $\mathrm{OR}$ staff in a virtual environment. Additionally, we have chosen to stay 1 week in each $O R$ to make healthcare workers to become familiar with the system and to minimise the Hawthorne effect behaviour modifications linked to the Hawthorne effect which will be evaluated by the collection of door opening one additional week in the absence of video camera. The results will be adjusted on this bias evaluation.

\section{INTRODUCTION}

Surgical site infection (SSI) is a major public health problem. It is the third most common healthcare-associated infection and contributes to $13-17 \%$ of all such infections. ${ }^{12}$ In 


\section{Strengths and limitations of this study}

- Second, the endpoints will not be the occurrence of the surgical site infection (SSI) but the 'pre-established best practices criteria' and surrogate of the infectious risk. The SSI rate would have been an ideally but unreachable endpoint. Indeed, obtaining a benchmarked SSI rate in these surgical units would have required a common protocol and a long duration of surveillance in clean surgical procedures with low SSI rates. In addition, SSI is multifactorial, and many confounding factors should have been collected. We will use the air and wound contamination as an indicator of infectious risk. There is no full correlation between these air contaminations and the occurrence of SSI but the link has been established several times in the past. Wound contamination occurs in $10-40 \%$ of surgical procedures, and fortunately rarely leads to SSI.

- Through this study, we will precisely and continually describe the dynamics and the perception of surgical staff during cardiac and orthopaedic surgery with the help of a motion tracking system. Data collected will help to understand the behaviours in the $\mathrm{OR}$ and their correlation with the infectious risk. This methodology will help to establish a detailed rule of the best practices and the good behavioural practices in the OR to prevent SSI.

France, the annual incidence of SSI rates varies from $0.6 \%$ to $8.8 \%$ according to the surgical specialty and contamination class. ${ }^{3}$ SSI substantially increases the severity of illness, prolongs the hospital length of stay, and increases mortality and costs. ${ }^{4-6}$ Preventive measures include skin preparation, surgical antibiotic prophylaxis in high-risk patients, control of the operating room (OR) environment and improvements in the surgical technique. Despite these measures, SSI still induces a substantial public health burden.

The risk factors for SSI are linked to the patient characteristics, including advanced age, diabetes, obesity and other comorbidities; the surgical procedures include notably contamination class, duration of operative procedure, surgeon's skill, control of hypothermia; and the OR environment and the postoperative stage. ${ }^{4}$ It is believed that the contamination of the surgical wound mainly occurs at the time of surgical procedure in the OR, eventually leading to SSI. Four main routes of microbial entry into an open clean surgical wound are usually described: (1) from the patient's skin, (2) from the surgeon's and other OR personnel, (3) by airborne microbes and (4) by instruments used during the surgical procedure. ${ }^{56}$ Wound contamination via haematogenous seeding from other source or postoperative wound contamination may occur, but seem rare sources of contamination. Controlling the OR environment with an appropriate ventilation system and discipline on the OR, with minimal movements is therefore critical for SSI prevention. $^{78}$

Humans naturally produce particles that could convey microorganisms. This phenomenon dramatically increases during displacements in the OR. ${ }^{9}$ The current guidelines do not include specific recommendations regarding the best OR staff behaviour (except for wearing cap, mask and scrub and performing appropriate hand hygiene) to decrease the exogenous risk of SSI. Some surgical or infection control societies advise to control the OR traffic in order to decrease air contamination and wound colonisation. ${ }^{10} 11$ These measures include limiting door opening and restricting the movements and the number of persons in the OR. However, these recommendations are often vague and are based on expert advice only without robust scientific arguments.

At the beginning of the $1980 \mathrm{~s}$, Lidwell et $a l^{12}$ evidenced a correlation between airborne contamination and wound contamination. Later, Tammelin et $a l^{13} 14$ demonstrated that surgical wound contamination could originate from the staff skin flora. Staff skin shedding could lead to spreading microorganisms by the air with occurrence of wound contamination..$^{915-17}$ In addition, the impact of the staff behaviour on SSI risks was assessed in several studies through the observation of the number of persons in the OR and the frequency of door opening. ${ }^{18} 19$ Overall, these studies were performed using classical audits with evaluation by human observers over short-time periods. This methodology does not allow the objective, continued and rigorous full collection of behaviour in the OR. Indeed, behaviours may change in the presence of an observer and observations may differ from one expert to another.

\section{Research questions}

To date, no study has described the global OR staff dynamics and behaviours during surgical intervention in the OR. This issue is challenged by the methodological concern of observational sessions by a direct observer. In consequence, the impact of the OR staff dynamics and behaviours during surgical intervention in the OR on the SSI risk has been partially assessed in previous studies. New technologies using motion capture systems appear as an alternative to obviate methodological issues. Automatic techniques based on sensors or motion capture allow the acquisition of objective data, with continued and prolonged periods of data collection. ${ }^{20} 21$

\section{Study objectives}

The present study will focus on the assessment and description of OR staff behaviours and on its association with the SSI risk during surgical procedures. This study aims at describing and assessing the staff behaviours in the OR and their variability by recording staff displacements using a motion tracking system and door opening detection system. Data obtained will be compared to the best practices previously established by an expert panel during an earlier part of the study. A secondary objective was to correlate the staff behaviours with the SSI risk, approached with surrogates of SSI, such as OR air contamination and wound contamination at the end of the surgical procedure. 
The main objective was to objectively measure the movements of surgical teams in the OR and to assess their adherence to the pre-established best practice criteria and their variability in a panel of ORs from two surgical specialties.

Secondary objectives are:

1. To assess correlations between movements of the OR personnel and the SSI risk, as approximated by surrogates of the infectious risk;

2. To assess the correlation between the particle count and the microbiological contamination in the OR air;

3. To describe the change in practices in the OR depending on the presence of a motion tracking system;

4. To assess the OR staff perception of their behaviour in the OR during an intervention to correlate with the actual data.

\section{METHODS AND ANALYSIS \\ Study design}

We propose an observational study based on the correlation between the data on OR behaviours obtained using new technology tools and (1) the 'best behaviour rules' established by an expert panel during an earlier part of the study and (2) surrogates of the infectious risk in the OR.

\section{Population and location of the study}

The study population will be formed of the OR personnel (surgeons, anaesthesiologists, nurses, nurse's aide) and any other person likely to enter the OR during the surgical procedure. Among this population, behaviours will be analysed by an automatic system of motion capture. Volunteers to participate will be selected by contacting the heads of the surgical and anaesthesiology teams and the infection control practitioner from each selected healthcare facility (HCF).

Surgical specialties and procedures have been included according to the following criteria: cutaneous approach, clean contamination class (Altemeier's class I), the frequency and the reproducibility of the procedure. On this basis, two specialties will be included: cardiac surgery with procedures requiring a full median sternotomy (planned coronary artery bypass grafting, valve repair or replacement surgery); and orthopaedic surgery for total hip and knee replacement.

\section{Strength evaluation}

The strength estimation for this comparative study depends on the variability of the staff behaviour between ORs. We hypothesise that the behaviour's variability is lower between the OR personnel in a same HCF than between two different HCFs. Thus, we have chosen to include a panel of HCFs to take into account this potential variability. We will include interventions occurring during half-day periods. For each surgical ward involved in the study, one OR will be randomly selected. The inclusion of 20 different ORs (10 in each specialty) will generate data for 50 cardiac procedures (1 patient per day) and for 50-100 orthopaedic procedures. The final analysis will be performed on a total of 100-150 procedures.

We will perform an observational multicentre study including 12 HCFs: 7 University hospitals, 1 semiprivate and 4 private hospitals located in France. Among the 12 participating HCFs, 10 OR of cardiac surgery and $10 \mathrm{OR}$ of orthopaedic surgery ( 7 public, 1 semiprivate and 2 private for both specialties) will be included in the study.

\section{Judgement criteria}

Primary criteria

Motion capture

The main judgement criteria will be the staff behaviour, as measured using his/her movements in the OR. A technology of motion capture based on a video tracking system will be adapted for the objective, continued and prolonged detection and the characterisation of movements in the OR. A network of eight video cameras (VICON-Bonita, Vicon, Los Angeles, USA) ${ }^{22}$ will be fixed upright to the wall by a suction system and linked by Ethernet cables to a hub. Data will be recorded on a laptop using the Vicon Tracker software (Vicon). Briefly, 68 LEDs situated on each camera produce an infrared light reflected by hemispherical markers and acquired by the optic. The detection of the same marker by different cameras allows its three-dimensional (3D) positioning.

The motion capture will be performed by a continuous tracking of reflective markers placed on the surgical caps/hoods of each person entering the OR. This system evaluates the movements of persons with a precision of $50 \mathrm{~cm}$. Four types of marker combinations will be created to distinguish four different professional categories: surgeon, anaesthesiologist (including anaesthesiology nurse and extracorporeal circulation personnel), OR nurse and other. The markers' positions are located in $3 \mathrm{D}$ by a method of spatial triangulation. The cartography of the OR including the situation of the table and doors will be performed at the system installation. Data recorded by the system will include the time and 3D position of the barycentre of each marker combination.

Doors opening will be collected by autonomous inertial sensors fixed on each door and synchronised to the motion tracking system. HiKoB FOX (HiKoB, Villeurbanne, France) is an autonomic system of wireless inertial sensors (tri-axial accelerometers, gyrometers and magnetometers). HiKoB FOX collects dynamic and door movements in real time. One sensor will be fixed on each door of the OR after a temporal synchronisation with the motion tracking system. The consistency between the position of persons in the OR and the doors traffic will be controlled through this synchronisation.

This device including the motion tracking system and the inertial sensors will stay during 1 week in the same OR to get people used to it and to take into account the 
potential behavioural modifications due to the Hawthorne effect. Data acquisition will start at patient entry in the OR and will continue until patient exit. Door opening sensors will be kept for one additional week to evaluate the impact of the Hawthorne effect on behaviour by comparing the frequency of door opening during and after removal of the motion tracking system.

\section{Best practices}

The best practices in the OR have been established using a Delphi method. During this procedure, we asked a French college of experts including five surgeons, five anaesthesiologists, five OR nurses and five infection control physicians, to quote a preselected list of behaviours and practices potentially linked to an increased SSI risk. These experts are professionals involved in surgical activities and are working daily in the field of infection control and SSI prevention. Overall, 24 experts were contacted and 20 agreed to participate. Items were preselected using the national and international guidelines for SSI prevention and were then discussed by a working group composed of one surgeon, one anaesthesiologist and three infection control physicians, all specialised in quality and safety in the OR. We selected 14 preoperative, 35 operative and 1 postoperative items. We asked to quote the infectious risk on a Likert scale varying from 1 (no impact on the infectious risk) to 9 (high impact on the infectious risk). Additionally, we asked to quote 14 additional items possibly collected by the motion capture system. An item was selected if quotations were higher than 6 for more than 17 participants.

Finally, 11 variables were considered to significantly increase the SSI risk. These variables were selected after two quotation rounds. Moreover, four parameters were considered to be interesting to collect with the motion tracking system (box 1).

\section{Secondary criteria}

Air sample

Microbiological air counts will be measured using an impactor air sampler (Air-test Omega, LCB, La Salle France) at a flow rate of $100 \mathrm{~L} / \mathrm{min}$ for $5 \mathrm{~min}(500 \mathrm{~L})$ sampling on to Trypticase soy agar (BioMerieux, France), which will then be incubated for 4 days at $30^{\circ} \mathrm{C}$. Air counts will be expressed as colony-forming units $/ \mathrm{m}^{3}$. The air sampler will be positioned at the head of the patient. After each sample, the impactor will be disinfected. Samples will be performed at the incision, every 30 min during orthopaedic surgery, every hour for cardiac surgery and at wound closing. The time of samples will be synchronised on the motion tracking computer clock.

\section{Particle count}

The particle count (HandiLaz Mini, Particle Measuring Systems, Boulder, USA) will be performed using a photo-detection device continuously from incision to
Box 1 The final criteria selected by a college of experts using a Delphi procedure

Parameters linked to an increase risk of surgical site infection (SSI)

Preoperative period

1. The knowledge and the actual implementation of the guidelines for SSI prevention.

2. Patient skin preparation (hair removal and skin antisepsis).

3. Setting up of sterile drapes.

4. Surgical hand disinfection of the surgical team.

5. Quality of air ventilation (type of flux, type of air contamination, pressures).

Operative period

6. Permanent wearing of scrub suits by every person in the operating room (OR) (mask/surgical caps).

7. Permanent wearing of specific sterile suits for the operating staff.

8. Wearing of a surgical cap covering all hair surfaces by all persons in the $\mathrm{OR}$ during the surgical procedure.

9. Wearing of a mask covering the nose and mouth by all persons in the OR during the surgical procedure.

10. Systematic replacement of material of wear in case of asepsis fault.

Postoperative period

11. Minimum time required for cleaning and refurnishing the $O R$ between two surgical procedures

Parameters interesting to collect using the video tracking system

1. Number of people in the $\mathrm{OR}$ during the surgical procedure.

2. Frequency of opening of $\mathrm{OR}$ doors.

3. Respect of the $\mathrm{OR}$ cleaning time between two interventions.

Limitation of the traffic in the $\mathrm{OR}$

wound closing. ${ }^{23}$ A base level of particle count will be performed for each OR at the beginning of the day before the staff entry. The particle analyser will sample 1 min every 2 min throughout the surgical procedure at a rate of $0.0283 \mathrm{~m}^{3} / \mathrm{min}\left(1.0 \mathrm{ft}^{3} / \mathrm{min}\right)$ and logged data at $1 \mathrm{~min}$ intervals to obtain the sample volumes of $0.0283 \mathrm{~m}^{3}(28.3 \mathrm{~L})$ of air. Samples will be collected through a $100 \mathrm{~cm}$ length of the surgical wound at the patient head. Particles will be classified by diameter (d) in six-size ranges: $0.3 \leq \mathrm{d}<0.5,0.5 \leq \mathrm{d}<1.0,1.0 \leq \mathrm{d}<3.0$, $3.0 \leq \mathrm{d}<5.0,5.0 \leq \mathrm{d}<10.0$ and $\mathrm{d} \geq 10 \mathrm{~mm}$. The count and particle size measurements will be recorded electronically by the particle analyser from the patient entry to the exit of the OR. The particle analyser will be synchronised on the motion tracking computer clock.

\section{Wound sampling}

A sample of the operating wound will be performed before closing. The sample method previously described by Tammelin et al, will use sterile pads of polyamide-polyester-viscose measuring $7.5 \times 7.5 \mathrm{~cm}$ placed on the subcutaneous tissue and removed after being soaked by wound liquids $(1 \mathrm{~min})$. This sample will be performed before any antiseptic aspersion. Microorganisms will be extracted by vortexing the pads during $2 \mathrm{~min}$ in a phosphate buffer saline (PBS with 
Tween 80 at $2 \%$ and lecithin at $0.3 \%$, Hyphen BioMed, Neuville sur Oise, France) inactivating antiseptics compounds. For each pad, an aliquot of $0.5 \mathrm{~mL}$ of PBS will be cultured on blood agar. Strains isolated will be quantified and identified by the investigator team using a mass spectrometry assay (MALDI-TOF-MS system, Microflex, Bruker Daltonics, Bremen, Germany). ${ }^{9}$

\section{Data collection}

Information will be collected on: (1) OR staff present during the intervention (age, gender, function, experience in the function, role in the surgical procedure), (2) surgical procedure (surgical specialty, surgical procedure, surgical technique used, incision time, preselected procedure periods, closure time), (3) surgical environment characteristics (air changes of filtered air per hour, positive pressure, temperature, relative humidity, particles contamination class, kinetic of particle decontamination class). The architecture of the OR will be taken into account by collection of the sizes and volumes of the room.

The motion tracking system will record all the behavioural parameters including: the number of persons in the OR, their proximity to the surgical theatre, the number of door openings, the number of exit/entry, the cumulated time in the OR and the interaction between the OR staff. These data will be stratified according to the professional categories.

As described above, particles' contamination will be continuously collected at the head of the patient from entry to exit from the OR. Microbiological data will include qualitative and quantitative values of the total air and wound flora.

Additionally, the perception of each OR staff will be collected using two different questionnaires: one assessing the safety climate, previously used by Sexton $e t a l^{21}$ and the other assessing the perception of each person regarding the infectious risk in his/her OR (figure 1). These questionnaires will be given to every OR team member and will assess six dimensions: perception of management, safety climate, stress recognition, job satisfaction, working conditions and teamwork climate. Participants will give his/her perception by rating 59 items corresponding to these six dimensions on a Likert scale going from A (disagree strongly) to $\mathrm{E}$ (agree strongly). The results will be stratified by the professional categories.

\section{Statistical analysis}

A descriptive analysis of all the parameters collected will be performed. For continuous variables (ie, age, duration of presence in the OR), indicators such as the mean, SD, minimum, median, quartiles and maximum values will be calculated.

$\chi^{2}$ Test will be used to evaluate the homogeneity between the observed behavioural data and the items of interest previously established by the college of experts, both categorical variables, with a level of significance for the $\mathrm{p}$ value fixed at $<0.05$. These analyses will be performed after stratification according to the surgical specialty and the type of HCF. Pearson or Spearman tests will be used to assess the correlation between the quantitative variables.

Additionally, a logistic regression will be used to independently analyse the link between the environmental variables (air wound contamination) and the behaviour variables all dichotomised according to the distribution of the population. The model will be adjusted according to the aerolic and architectural characteristics of the OR. The model will also be adjusted according to the Hawthorne effect with the comparison of door opening data obtained with and without the video tracking system.

Finally, a multilevel model will allow including contextual variation due to the surgical specialty or the type of HCF. The method will quantify the 'surgical specialty' effect and the 'HCF type' effect.

\section{Confidentiality issue}

The motion capture system will not allow identifying people who are symbolised by markers on the head cap. Functions of the OR team members will be collected but no name data will be recorded. The system will be presented to surgical and anaesthesiology teams in each participating centre and OR. Included patients will systematically be informed by an information letter. Additionally, this methodology requires the consent of the OR members included in the study.

\section{DISCUSSION}

The operating theatre is a particular area in the hospital. This special care environment with sophisticated techniques generates several ranges of risks for the patient including the occurrence of infection. Several recommendations have been published (skin preparation, surgical antibiotic prophylaxis, control of the OR environment and improvements in the surgical technique) to improve the patient safety and quality of care in the OR..$^{10} 112425$ Most of the recommendations are based on the scientific evidence. However, guidelines for the prevention of transmission of microorganisms to the surgical wound and eventually to SSI are scarce and often fuzzy. The existing recommendations are based on expert advice only without any scientific proof of evidence. In consequence, rituals abound in operating departments to prevent environmental contamination and impact staff behaviour. The present study will aim to bring a rationale for the prevention of airborne microorganism transmission by the description of best behaviour rules in the OR.

The impact of behaviours on the SSI risk has been studied in the past. We performed a search on the MEDLINE database in March 2013 and found eight articles assessing the correlation between the behaviour and the infectious risk in the OR. Results were conflicting. Among those, five articles studied the traffic flow by 
Figure 1 Questionnaire to assess the perception of each surgical staff member regarding the infections risk in his/her operating room.
ARIBO study:

Perception of the infectious risk in the Operating room

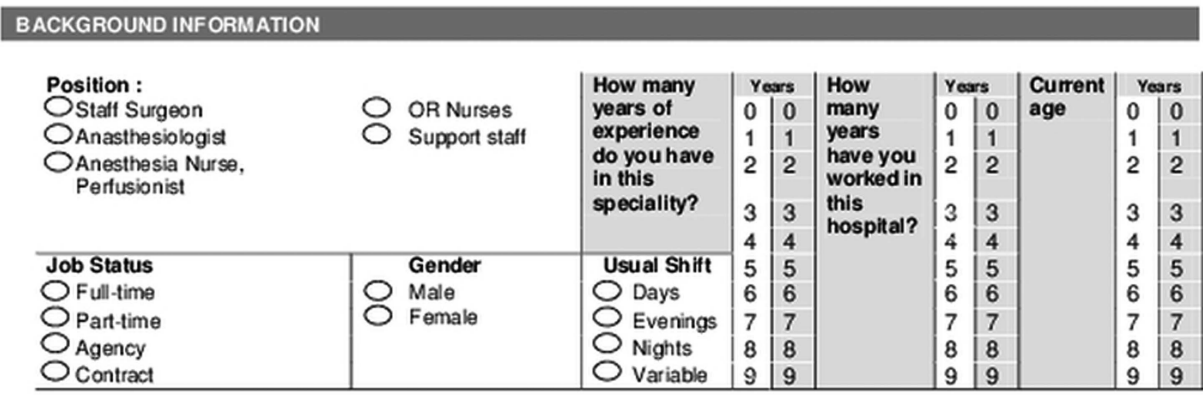

Please answer by marking the response of your choice to the right Of each item, using

\begin{tabular}{|c|c|c|c|c|c|}
\hline A & B & C & D & E & X \\
\hline $\begin{array}{c}\text { Disagree } \\
\text { strongly }\end{array}$ & $\begin{array}{c}\text { Disagree } \\
\text { strongly }\end{array}$ & Neutral & $\begin{array}{c}\text { Agree } \\
\text { Sllightly }\end{array}$ & $\begin{array}{c}\text { Agree } \\
\text { strongly }\end{array}$ & $\begin{array}{c}\text { Not } \\
\text { applicable }\end{array}$ \\
\hline
\end{tabular}

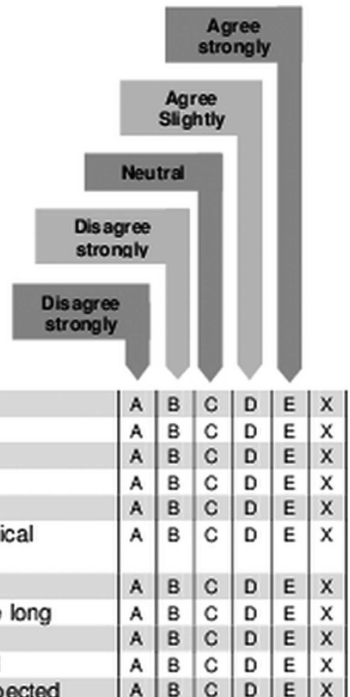

evaluating the number of door openings and reason for door opening and the number of persons attending the OR during the intervention. Four of them were only descriptive studies and the last study correlated the traffic with the air contamination as a surrogate of the SSI risk. ${ }^{18} 19$ 26-28 These studies were based on human observations performed at a given time. Collecting information using observers may have induced two biases: the Hawthorne effect and the partial observation of dynamics in the OR.

In two other articles, a bundle of preventive measures (including behavioural measures) were implemented and showed some an impact on the SSI rates. ${ }^{89}$ Finally, a study evaluated the impact of noise and found a strong positive correlation between the increase in decibels and the SSI rates. ${ }^{30}$ These studies were based on human observations of door opening or person present in the OR at given times. However, no study systematically and continuously evaluated a global perception of the staff dynamics in the OR.

Through the present study, we will develop an original approach using high technology tools (motion tracking and inertial sensors) to evaluate the behavioural dynamics of healthcare workers in the OR and their impact on the SSI risk. Other studies have used video to audit practices in the OR. ${ }^{31}$ This system was generally used to analyse and improve the performance of surgical techniques and to prevent an adverse event in the OR. ${ }^{32} 33$ This type of recording system has also been used to improve practices outside the OR. A study showed that compliance with hand hygiene could increase from less than $10-86 \%$ due to the presence of remote video auditing. ${ }^{20}$

This challenging project gathers specialists from several disciplines (infection control, epidemiology, surgery, anaesthesiology, psychology and engineering) and will allow obtaining the qualitative and quantitative epidemiological data. This consistent approach will allow collecting data that will help to understand the behavioural origin of the SSI risk and to improve the quality of care in the OR.

\footnotetext{
Author affiliations

${ }^{1}$ IAME, UMR 1137, Univ Paris Diderot, Sorbonne Paris Cité, Paris, France

${ }^{2}$ IAME, UMR 1137, INSERM, Paris, France

${ }^{3}$ Infection Control Unit, AP-HP, Hôpital Bichat, Paris, France

${ }^{4}$ Laboratoire d'Informatique de Robotique et de Microélectronique de Montpellier (LIRMM), CNRS: UMR5506-Université Montpellier II-Sciences et techniquesNRIA Sophia Antipolis-LIRMM, Montpellier, France
} 
${ }^{5}$ Institut National de Recherche en Informatique et en Automatique, Montbonnot, France

${ }^{6}$ Infection Control Unit, Lille University Hospital, Lille, France

${ }^{7}$ Laboratoire de I'Informatique du Parallélisme (LIP), PRES Université de Lyon

—CNRS: UMR5668—Ecole Normale Supérieure-Lyon, Université Claude

Bernard, Lyon I, France

Acknowledgements The authors would like to thank Professor H Migaud and Professor Vincent Piriou for their help in sectioning Delphi items and all experts for their participation in the Delphi procedure to establish the best practices criteria: Infection control physicians: J Hajjar, A Chalfine, B Jarrige, JC Seguier, P Berthelot; operating room/infection control nurses: L Cauchy, AC Guille des Buttes, C Maertens, R Leservoisier, M Aggoune; surgeons: JP Mignard, B Barry, JL Jost, 0 Farges, L Richard; anaesthetists: MP Dilly, B Abry, R Dumont, S Molliex, P Albaladejo.

Contributors CA, BG, GT, RP-G, EF, J-CL and GB have been involved in drafting the manuscript and have made contributions to the conception and design of the study.

Funding This study was partly supported by the French Ministry of Health (national grant PREQHOS 2011).

Competing interests None.

Ethics approval This protocol was approved by the Institutional Review Board of the (IRB) of Paris North Hospitals, Paris 7 University, AP-HP (no 11-113, 6 April 2012). The findings of the study will be disseminated through peer-reviewed journals, national and international conference presentations.

Provenance and peer review Not commissioned; internally peer reviewed.

Open Access This is an Open Access article distributed in accordance with the Creative Commons Attribution Non Commercial (CC BY-NC 3.0) license, which permits others to distribute, remix, adapt, build upon this work noncommercially, and license their derivative works on different terms, provided the original work is properly cited and the use is non-commercial. See: http:// creativecommons.org/licenses/by-nc/3.0/

\section{REFERENCES}

1. Anderson DJ, Kaye KS, Classen D, et al. Strategies to prevent surgical site infections in acute care hospitals. Infect Control Hosp Epidemiol 2008;29(Suppl 1):S51-61.

2. European Center for Disease Prevention and Control. Annual epidemiological report on communicable diseases in Europe. 2012. http://www.ecdc.europa.eu/en/publications/Publications/AnnualEpidemiological-Report-2012.pdf?bcsi scan 628cd39dca2568d2= FnAPsXbe9HUkDWZzifxo42fFriUBAAAAVj/yAQ==\&bcsi_scan_ filename=Annual-Epidemiological-Report-2012.pdf

3. Raiseau d'alerte d'investigation et de surveillance des infections nosocomiales. Surveillance des infections du site opératoire. 2011. http://www.cclinparisnord.org/Inciso/RappRAISIN2011.pdf

4. Barie PS, Eachempati SR. Surgical site infections. Surg Clin North Am 2005;85:1115-35, viii-ix.

5. Mastro TD, Farley TA, Elliott JA, et al. An outbreak of surgical-wound infections due to group A streptococcus carried on the scalp. $N$ Engl J Med 1990;323:968-72.

6. Dineen P, Drusin L. Epidemics of postoperative wound infections associated with hair carriers. Lancet 1973;2:1157-9.

7. Gastmeier P, Breier AC, Brandt C. Influence of laminar airflow on prosthetic joint infections: a systematic review. J Hosp Infect 2012;81:73-8

8. Beldi G, Bisch-Knaden S, Banz V, et al. Impact of intraoperative behavior on surgical site infections. Am J Surg 2009;198:157-62.

9. Mackintosh CA, Lidwell OM, Towers AG, et al. The dimensions of skin fragments dispersed into the air during activity. J Hyg (Lond) 1978;81:471-9.

10. Mangram AJ, Horan TC, Pearson ML, et al. Guideline for prevention of surgical site infection, 1999. Hospital Infection Control Practices
Advisory Committee. Infect Control Hosp Epidemiol 1999;20: 250-78; quiz 79-80.

11. Société Francaise d'Hygiène Hospitalière. La qualité de l'air au bloc opératoire. 2004.

12. Lidwell OM, Lowbury EJ, Whyte W, et al. Effect of ultraclean air in operating rooms on deep sepsis in the joint after total hip or knee replacement: a randomised study. BMJ 1982;285:10-4.

13. Tammelin A, Hambraeus A, Stahle E. Source and route of methicillin-resistant Staphylococcus epidermidis transmitted to the surgical wound during cardio-thoracic surgery. Possibility of preventing wound contamination by use of special scrub suits. $J$ Hosp Infect 2001;47:266-76.

14. Tammelin A, Hambraeus A, Stahle E. Routes and sources of Staphylococcus aureus transmitted to the surgical wound during cardiothoracic surgery: possibility of preventing wound contamination by use of special scrub suits. Infect Control Hosp Epidemiol 2001;22:338-46.

15. Verkkala K, Eklund A, Ojajarvi J, et al. The conventionally ventilated operating theatre and air contamination control during cardiac surgery-bacteriological and particulate matter control garment options for low level contamination. Eur J Cardiothorac Surg 1998;14:206-10.

16. Hambraeus A. Aerobiology in the operating room-a review. J Hosp Infect 1988;11(Suppl A):68-76.

17. Noble WC, Lidwell OM, Kingston D. The size distribution of airborne particles carrying micro-organisms. J Hyg (Lond) 1963;61:385-91.

18. Lynch RJ, Englesbe MJ, Sturm L, et al. Measurement of foot traffic in the operating room: implications for infection control. Am J Med Qual 2009;24:45-52.

19. Young RS, O'Regan DJ. Cardiac surgical theatre traffic: time for traffic calming measures? Interact Cardiovasc Thorac Surg 2010;10:526-9.

20. Armellino D, Hussain E, Schilling ME, et al. Using high-technology to enforce low-technology safety measures: the use of third-party remote video auditing and real-time feedback in healthcare. Clin Infect Dis 2012;54:1-7.

21. Lucet JC, Laouenan C, Chelius G, et al. Electronic sensors for assessing interactions between healthcare workers and patients under airborne precautions. PLOS ONE 2012;7:e37893.

22. Isableu B, Hansen $\mathrm{C}$, Rezzoug N, et al. Velocity-dependent changes of rotational axes during the control of unconstrained 3D arm motions depend on initial instruction on limb position. Hum Mov Sci 2013;32:290-300.

23. Dharan S, Pittet D. Environmental controls in operating theatres. $J$ Hosp Infect 2002;51:79-84.

24. National Collaborating Centre for Women's and Children's Health. Surgical site infection: prevention and treatment of surgical site infection. 2008.

25. Société Francaise d'Hygiène Hospitalière. Conference de consensus: 'Gestion pre-operatoire du risque infectieux'. 2004.

26. Panahi $P$, Stroh $M$, Casper DS, et al. Operating room traffic is major concern during total joint arthroplasty. Clin Orthop Relat Res 2012;470:2690-4.

27. Parikh SN, Grice SS, Schnell BM, et al. Operating room traffic: is there any role of monitoring it? J Pediatr Orthop 2010;30:617-23.

28. Andersson AE, Bergh I, Karlsson J, et al. Traffic flow in the operating room: an explorative and descriptive study on air quality during orthopedic trauma implant surgery. Am J Infect Control 2012;40:750-5.

29. Borer A, Gilad J, Meydan N, et al. Impact of active monitoring of infection control practices on deep sternal infection after open-heart surgery. Ann Thorac Surg 2001;72:515-20.

30. Kurmann A, Peter M, Tschan F, et al. Adverse effect of noise in the operating theatre on surgical-site infection. Br J Surg 2011;98:1021-5.

31. Makary MA. The power of video recording: taking quality to the next level. JAMA 2013;309:1591-2.

32. Rex DK, Hewett DG, Raghavendra M, et al. The impact of videorecording on the quality of colonoscopy performance: a pilot study. Am J Gastroenterol 2010;105:2312-17.

33. Jacobs B, Duncan JR, Street $M$, et al. Audio and video recording system for routine documentation of fluoroscopic procedures. $J$ Vasc Interv Radiol 2010;21:725-9. 